\title{
Evaluation of External Root Resorption of Endodontically Treated and Vital Teeth during Orthodontic Treatment: A Systematic Review
}

\author{
Goda Bilvinaitė, Greta Patkauskaitè $\dot{e}^{*}$ and Vilija Berlin \\ Faculty of Medicine, Institute of Dentistry, Vilnius University, Lithuania
}

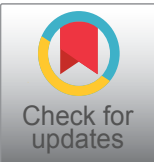

*Corresponding author: Greta Patkauskaite, Faculty of Medicine, Institute of Dentistry, Vilnius University, Vilnius, Lithuania

Goda Bilvinaite, Faculty of Medicine, Institute of Dentistry, Vilnius University, Vilnius, Lithuania

\begin{abstract}
Background: External root resorption is a common side effect of orthodontic treatment. Although endodontically treated teeth respond similarly to vital teeth to the application of force during orthodontic therapy, studies show conflicting results. Researcher's present different results that endodontically treated teeth are less affected, equally affected, or more affected than vital teeth. It is important for clinicians to know whether teeth treated with endodontics exhibit more, less or the same amount of root resorption in order to ensure a successful orthodontic treatment without complications.
\end{abstract}

Methods: This systematic review evaluated root resorption of endodontically treated and vital teeth as an outcome for patients who underwent orthodontic treatment. Electronic databases (PubMed, MEDLINE, Wiley, Clinical Key, Web of Science, ScienceDirect, Scopus, and Cochrane) were searched. Study selection criteria included randomized controlled clinical trials (RCTs), non-randomized controlled clinical trials, prospective or retrospective controlled clinical trials (CCTs). Two authors independently reviewed evaluated and extracted data from the selected studies. Analysis of literature was performed using the PRISMA analysis protocol.

Results: 10 out of 1,814 original papers met the inclusion criteria. A total of 351 patients were included in these studies. More than half of the studies present that there are no significant differences between the amount of root resorption between endodontically treated and vital teeth. Other studies evidence suggests that endodontically treated teeth are more resistant to external root resorption compared to their vital contralateral teeth.

Conclusions: Orthodontic treatment does not increase root resorption for endodontically treated teeth, the amount of resorption is the same as vital teeth or smaller.

\section{Keywords}

External root resorption, Orthodontic treatment, Endodontically treated teeth, Vital teeth

\begin{abstract}
Abbreviations
OIIRR: Orthodontically Induced Inflammatory Root Resorption; RCTs: Randomized controlled clinical; CCTs: Controlled Clinical Trials; CBCT: Cone Beam Computed Tomography; micro-CT: micro-Computed Tomography; M-CSF: Macrophage Colony Stimulating Factor; RANKL: Receptor Activator of Nuclear Factor Kappa-B Ligand; IL-1 $\beta$ : Interleukin-1 $\beta$; TNF- $\alpha$ : Tumor Necrosis Factor Alpha
\end{abstract}

\section{Introduction}

Root resorption is described as a complicated, non-reversible pathologic process, which affects the external layers of the root or the apex [1]. It is a sterile, locally inflamed process and has all characteristic symptoms of inflammation. External root resorption is a common side effect of orthodontic treatment. Small changes in the length of root and thickness of cement and dentine are noticed in almost every orthodontic treatment, whereas more severe resorption is documented to be in around 1 to 5\% [2]. Wasserstein introduced the term orthodontically induced inflammatory root resorption (OIIRR) to differentiate this type of resorption from others such as those caused by trauma, periapical lesions of periodontal disease [3]. The degree and severity of the external root resorption associated with orthodontic treatment depends on several factors, some

Citation: Bilvinaitė G, Patkauskaitė G, Berlin V (2021) Evaluation of External Root Resorption of Endodontically Treated and Vital Teeth during Orthodontic Treatment: A Systematic Review. Int J Oral Dent Health 7:128. doi.org/10.23937/2469-5734/1510128

Accepted: April 19, 2021: Published: April 21, 2021

Copyright: (C) 2021 Bilvinaitè G, et al. This is an open-access article distributed under the terms of the Creative Commons Attribution License, which permits unrestricted use, distribution, and reproduction in any medium, provided the original author and source are credited. 
of which are general (genetics, age, systemic diseases, type of teeth) and mechanical (magnitude and type of orthodontic force, type of tooth movement) [4]. However, some of the factors still have controversial results. There are numerous clinical trials carried out analyzing the effect of orthodontic treatment on endodontically treated teeth, however the results differ. Some authors express that endodontically treated teeth are less affected [5], equally affected [6], or more affected than vital teeth [7]. The evidence on resorptions of endodontically treated teeth when moved orthodontically shows conflicting results and needs to be re-evaluated. This article aims to investigate the currently existing clinical trials to figure out, whether there is a significant difference between the resorption of endodontically treated teeth and vital teeth caused by orthodontic forces.

\section{Materials and Methods}

\section{Data sources and searches}

Analysis of literature was performed using the PRISMA analysis protocol. A comprehensive search was conducted by using electronic databases as well as by manual search to identify all relevant studies related to orthodontic-induced root resorption of vital and endodontically treated teeth. PubMed, Wiley, Scopus, Cochrane Library and ScienceDirect electronic databases were used. The search covered all articles published from January 2000 to December 2020 by using the following key words: ["resorption" AND ("endodontic" OR "root-filled") AND "vital" AND "orthodontic" ].

\section{Study selection and data extraction}

Two independent reviewers were involved in screening of titles, abstracts and full-text reports. Inter- reviewer agreement on study selection and data extraction was calculated by using Cohen's kappa. In cases of disagreement, the reviewers discussed to reach a common final decision, a third reviewer was involved if necessary.

In the first step, titles and abstracts were screened for relevance by applying the inclusion and exclusion criteria. The inclusion criteria included the following:

- Randomized controlled clinical trials (RCTs), non-randomized controlled clinical trials, prospective or retrospective controlled clinical trials (CCTs).

- Studies published in English with full text available.

- Studies performed on humans.

- Studies evaluating the incidence and the severity of root resorption between endodontically treated and vital teeth as an outcome of orthodontic treatment.

The exclusion criteria were:
- Animal studies.

- Case reports or series.

- Literature reviews.

- Studies involving only endodontically treated or vital teeth.

To avoid bias, any studies identified by either one reviewer during this initial screening were included. In the second step, full texts of selected studies were read for assessment of eligibility. Studies that were selected at this second-stage screening were submitted for data extraction. The following information was extracted for each included study: study characteristics (author, year of publication, study design, method of evaluation), treatment characteristics (treatment type, treatment duration), patients' characteristics (number of patients, mean age, teeth type) and outcome characteristics (resorption type and severity). The authors were contacted in cases of any missing or unpublished essential information.

\section{Quality assessment}

The quality of the included studies was evaluated by two observers independently according to a scoring system used by Gordon and colleagues [8]. The original scoring system was minimally changed and adapted to our systematic review. Table 1 presents the criteria of methodologic scoring system. The methodologic quality was determined by percentages of the maximum achievable score (14 points): Mean score $<60 \%$ indicated poor quality; $60 \%$ to $70 \%$-moderate quality; >

Table 1: Methodologic scoring system.

\section{Study design (maximum score-7):}

\begin{tabular}{|l|l|}
\hline A. Objective & 1 Point if clearly formulated \\
\hline B. Sample size & 1 Point if more than 15 \\
\hline C. Sample evaluation & $\begin{array}{l}\text { 1 Point if estimated before } \\
\text { collection of data }\end{array}$ \\
\hline D. Selection criteria & 1 Point if clearly described \\
\hline E. Study time & 1 Point if prospective \\
\hline F. Randomization & 1 Point if stated \\
\hline G. Control group & 1 Point if present \\
\hline
\end{tabular}

\section{Study measurements (maximum score-3)}

\begin{tabular}{|l|l|}
\hline H. Measurement method & 1 Point if clearly described \\
\hline I. Blind measurement & 1 Point if stated \\
\hline J. Resorption detection & $\begin{array}{l}\text { 1 Point if radiographs, CBCT } \\
\text { or micro-CT }\end{array}$ \\
\hline
\end{tabular}

\section{Statistical analysis (maximum score-4)}

K. Statistical analysis method 1 Point if appropriate

\begin{tabular}{|l|l|}
\hline L. Error of method & 1 Point if stated \\
\hline $\begin{array}{l}\text { M. Statistical significance } \\
\text { level }\end{array}$ & 1 Point if $p$ value stated \\
\hline N. Data presentation & $\begin{array}{l}\text { 1 Point if any variability } \\
\text { measures stated }\end{array}$ \\
\hline
\end{tabular}




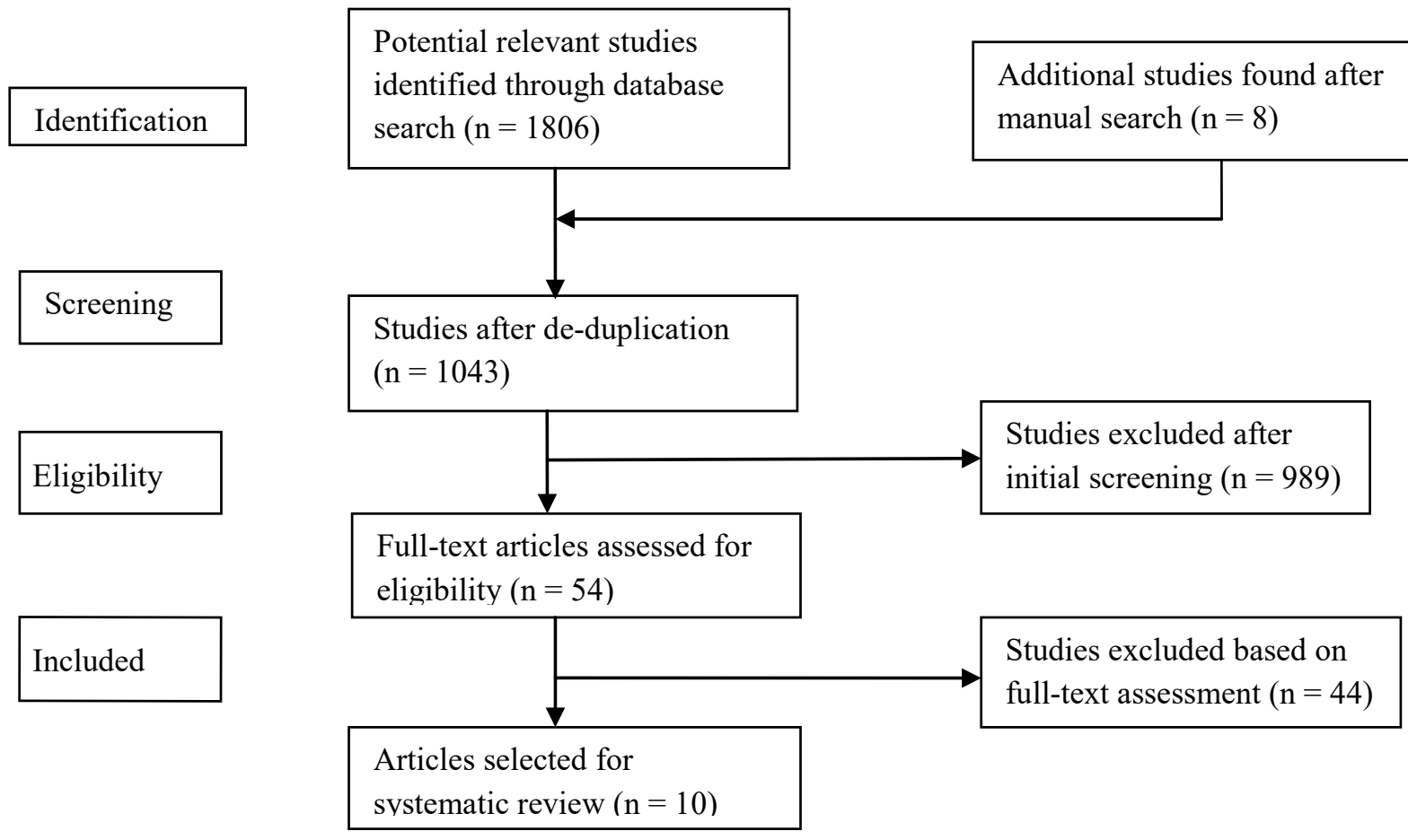

Figure 1: Flow diagram for the identification and selection of studies in this systematic review.

70\% -good quality. Inter-viewer agreement on quality assessment was evaluated by Cohen's kappa.

\section{Results}

\section{Study selection}

The flowchart of the study is demonstrated in Figure 1. The initial electronic and manual searches yielded 1814 studies that were reduced to 1043 studies after subtraction of duplicates. During the screening phase, 989 articles were regarded as irrelevant or did not fulfil the inclusion criteria, according to titles and abstracts. The full text of the remaining 54 articles were reviewed to evaluate the eligibility of each study and only 10 articles in total were qualified for this systematic review. The excellent agreement between two reviewers during the selection of the studies was confirmed by the Cohen's kappa of 0.941 .

\section{Study characteristics}

All studies involved in this systematic review were retrospective $(n=7)$ or prospective $(n=3)$ controlled clinical trials. The characteristics of included studies are summarized in Table 2. The Cohen's kappa score of 1.000 indicated a perfect inter-reviewer agreement for data extraction.

A total of 351 patients with mean ages ranging between 12.8 and 39 years ( 2 articles did not mention the age) were included in these studies. Endodontically treated teeth and their contralateral vital teeth were evaluated before and after treatment by using digital panoramic radiographs $(n=6)$, periapical radiographs $(n=2)$, cone beam computed tomography (CBCT) $(n=$ 1) or combination of radiographs and micro-computed tomography (micro-CT) $(n=1)$. All selected studies used the bracket system for orthodontic therapy. The duration of treatment varied from 8 weeks to 3.17 years. The majority of studies $(n=9)$ assessed external apical root resorption by measuring the tooth axial length from the root apex to corresponding incisal or occlusal edge while one study calculated the volume of external root resorption separately in the cervical, middle and apical thirds. The amount of resorption was evaluated at various types of teeth with the exclusion of third molars. Regarding the results of six involved studies, no statistically significant differences were observed between endodontically treated teeth and their contralateral vital teeth $(p>0.05)$. Other studies $(n=4)$ determined vital teeth to be affected by root resorption significantly more in comparison to root-filled teeth $(p<0.05)$.

The Cohen's kappa score of 0.830 indicated almost perfect inter-reviewer agreement on quality assessment. The overall quality of the studies involved for this systematic review was evaluated as "Good" as methodologic quality scores ranged from $71.4 \%$ to $85.7 \%$ of the maximum achievable score (mean score $77.9 \%$ ).

\section{Discussion}

The main goal of this systematic review was to obtain reliable evidence comparing external root resorption of endodontically treated and vital teeth caused by orthodontic treatment. The clinical relevance of this subject should be highlighted because the orthodontist should be aware of expectations about possible outcomes during orthodontic treatment that could cause serious consequences. After a comprehensive literature search and evaluation, numerous articles were eliminated be- 


\begin{tabular}{|c|c|c|c|c|c|c|c|c|c|c|}
\hline$\frac{n}{5}$ & 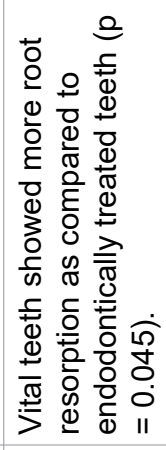 & 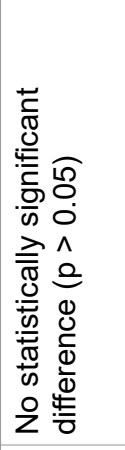 & 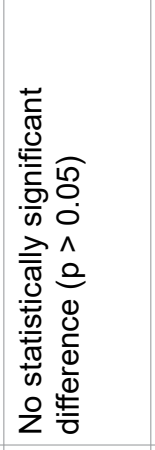 & 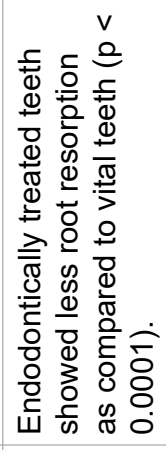 & 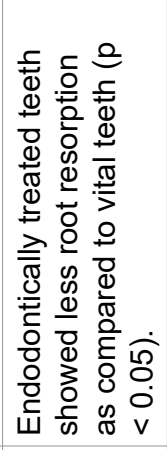 & 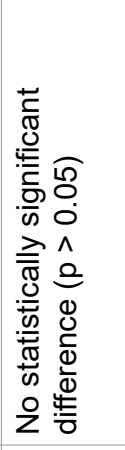 & 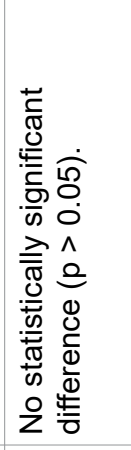 & 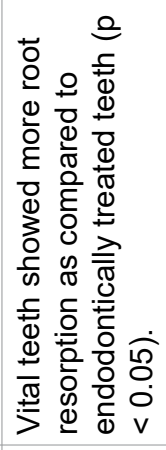 & 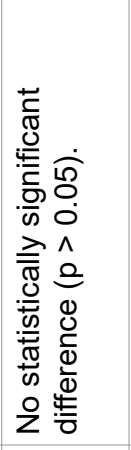 & 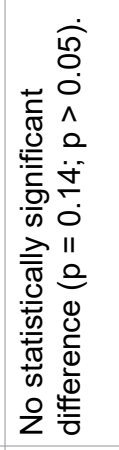 \\
\hline 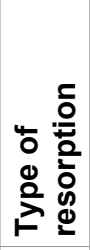 & 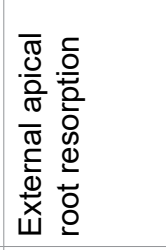 & 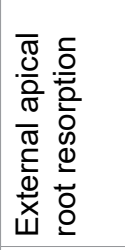 & 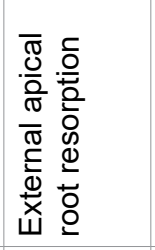 & 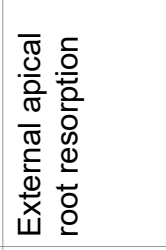 & 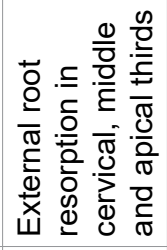 & 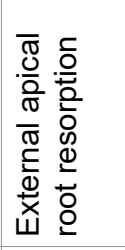 & 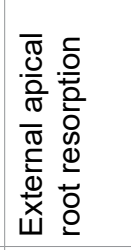 & 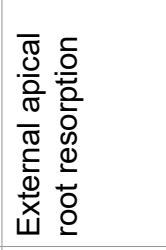 & 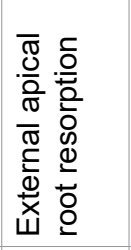 & 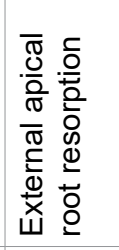 \\
\hline 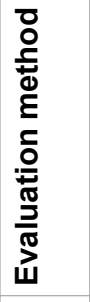 & 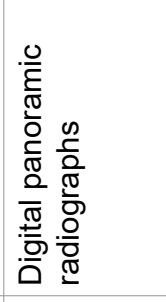 & 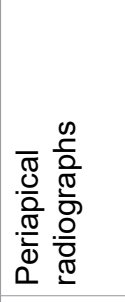 & 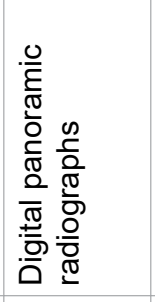 & 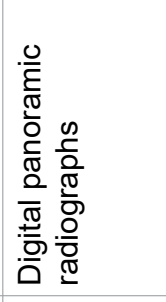 & 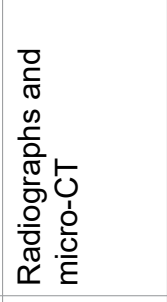 & $\begin{array}{l}\llcorner \\
\text { ' }\end{array}$ & 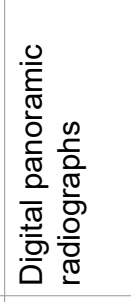 & 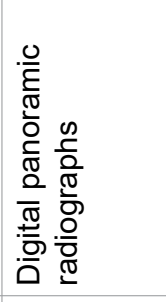 & 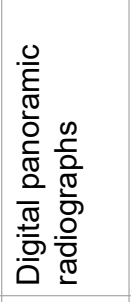 & 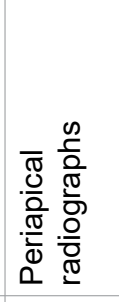 \\
\hline 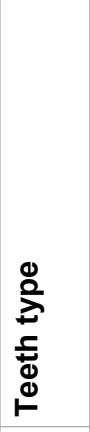 & 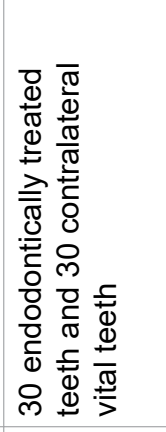 & 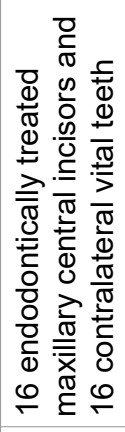 & 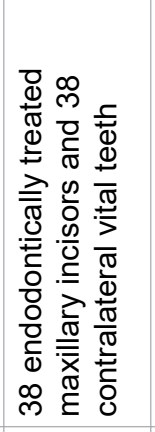 & 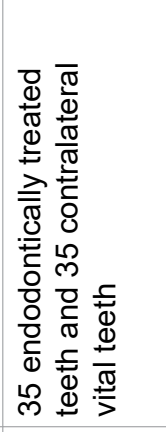 & 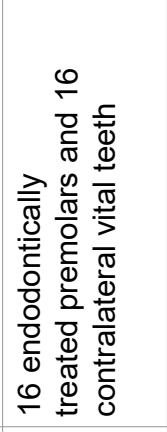 & 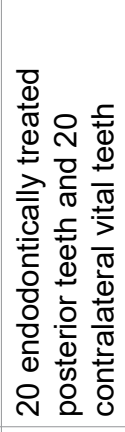 & 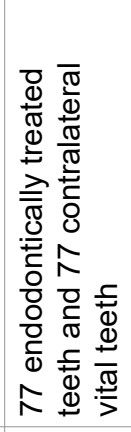 & 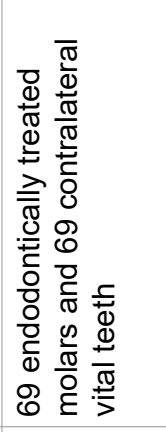 & $\begin{array}{l}\frac{0}{4} \\
0 \\
\Phi \\
0 \\
0 \\
0 \\
0\end{array}$ & 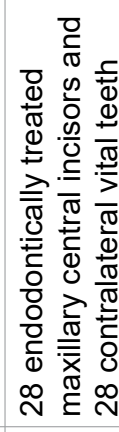 \\
\hline 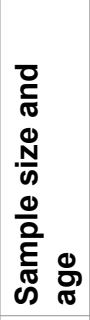 & 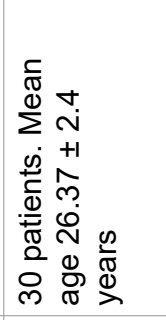 & 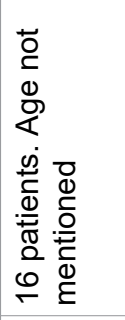 & 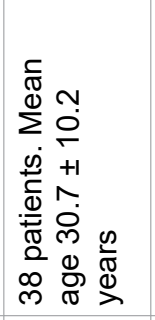 & 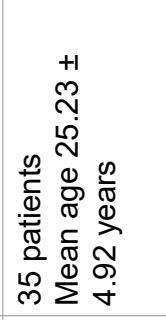 & 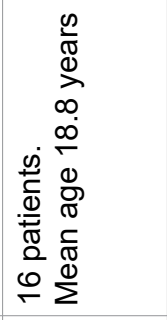 & 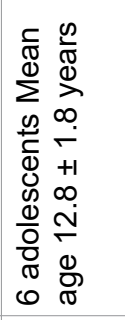 & 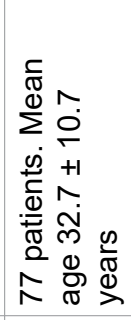 & 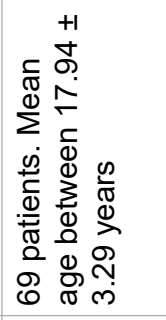 & 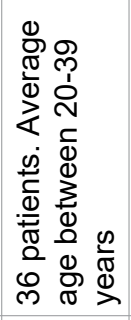 & 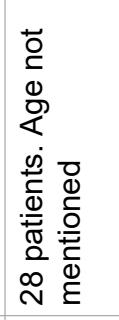 \\
\hline 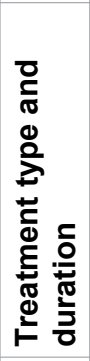 & 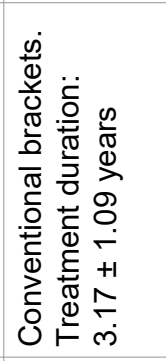 & 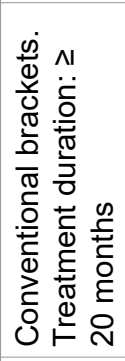 & 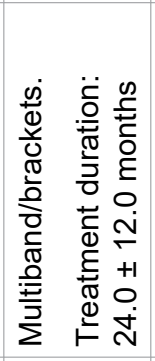 & 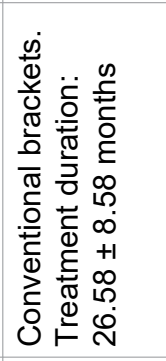 & 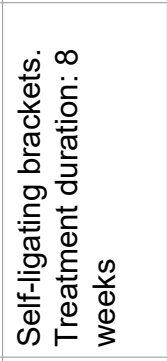 & 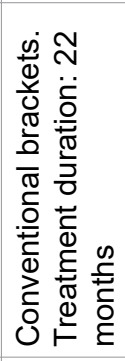 & 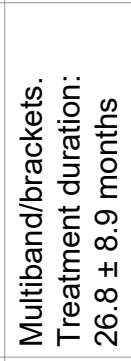 & 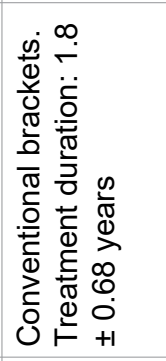 & 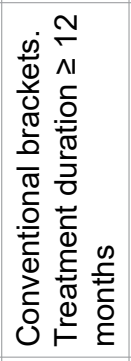 & 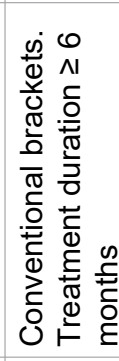 \\
\hline 竞 & 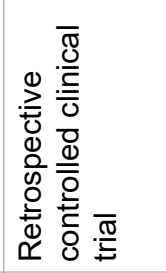 & 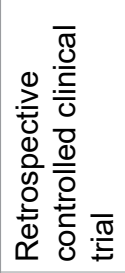 & 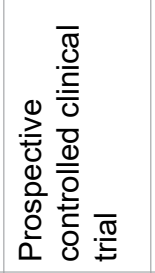 & 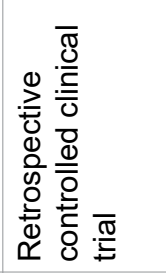 & 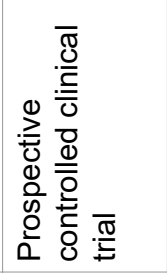 & 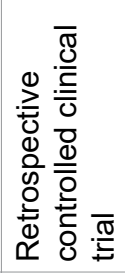 & 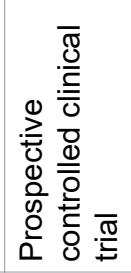 & 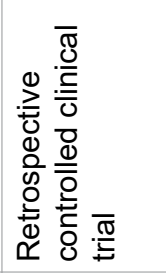 & 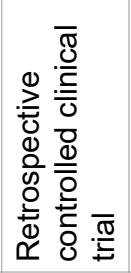 & 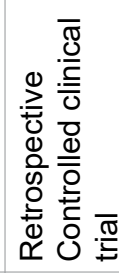 \\
\hline 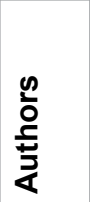 & 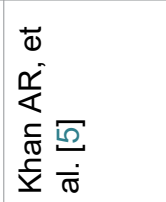 & 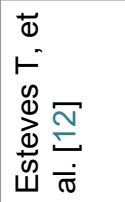 & 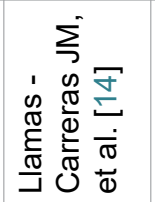 & 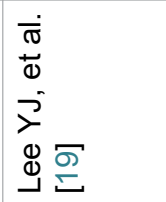 & 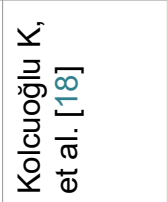 & 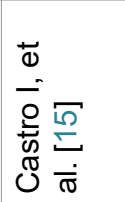 & 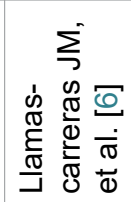 & 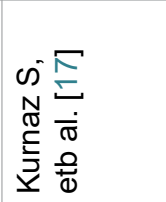 & 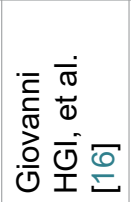 & 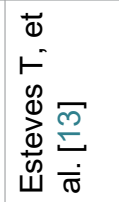 \\
\hline
\end{tabular}


cause of a precise search protocol and a list of criteria that all articles had to meet. After evaluation of every article only 10 of them were recruited in this systematic review and were analysed further.

In the past, few studies indicated that endodontically treated teeth are more prone to resorption compared to vital teeth. Steadman examined histological changes of endodontically treated teeth and stated that the root filling can act as a foreign body and cause chronic irritation, therefore determined to be more vulnerable to resorption [9]. It is necessary to mention that this can only happen in cases of overfilled teeth when the filling passes through the apical foramen and ends up in the periapical region. This reaction is not usually observed in teeth that are filled correctly. Wickwire NA, et al. in the article published in 1974 stated that endodontically treated teeth showed a greater frequency of root resorption compared to the vital pulp contralateral teeth [10]. However, this article has been widely discussed and proven to be misleading. Their methods involved teeth that were treated endodontically after trauma, which could increase their susceptibility to resorption [11]. In an animal study carried out by Mah, et al. the results showed greater loss of cement for endodontically treated teeth compared to the vital group [7]. Nonetheless, the radiographs did not show a statistically significant difference of root resorption between the two observed groups. All of these mentioned studies, that have been done in the $20^{\text {th }}$ century, exhibit numerous disadvantages and were not included in the systemic review, however, their contribution and conclusions lead to creation of further research and the results that are widely accepted today.

Careful analysis of selected studies evidenced that there are differences between the results of chosen studies. 6 out of 10 studies have shown no significant differences in root resorption between vital and rootfilled teeth. In a study carried out by Esteves, et al. the results showed that $50 \%$ of endodontically treated teeth had a greater root resorption compared to the vital group, however the other $50 \%$ showed more resorption in the vital teeth group [12]. The authors also mention that all teeth show some degree of resorption during orthodontic tooth movement. It is necessary to mention that the examined sample of patients is usually considered small to present firm conclusions. In another study by Esteves, et al. the results present the same outcome [13]. They measured that $42.8 \%$ of patients had greater root resorption in the endodontically treated teeth compared to the vital teeth group. However, the other $51.2 \%$ showed greater resorption in vital teeth. The small differences between the groups in both of the studies conclude that there is no significant difference between them. A prospective study by Llamas Carreras, et al. revealed that $48 \%$ of patients had a greater root resorption in endodontically treated teeth compared to the control group, however in the total sample it showed to be statistically insignificant [6]. This study lacks means of measurements and reports only the resorption proportions, which could be considered and incomplete outcome data. Llamas Carreras, et al. carried out another study and the reached results present the same answer [14]. They found out that $68.4 \%$ of patients had a greater resorption of endodontically treated teeth, and $31.6 \%$ of patients it was the vital teeth group that showed a greater resorption. However, the standard deviation indicated that in the total sample there are no significant differences between the level of root resorption by comparing both groups. A study by Castro, et al. is in agreement with the studies mentioned above [15]. No previous study had used the $\mathrm{CBCT}$ to analyze the changes of roots during orthodontic treatment, however, the results support the finding that orthodontic force affects vital and root filled teeth similarly mildly. The mean resorption in endodontically treated teeth $(-0.30 \mathrm{~mm})$ was considerably greater than that of the vital group $(-0.16 \mathrm{~mm})$ although no statistical difference was noticed. Giovanni and Eliezer present results that show higher root resorption in endodontically treated teeth $(0.84 \pm 0.48 \mathrm{~mm})$ and lower in vital teeth $(0.78 \pm 0.42 \mathrm{~mm})$ [16]. After completing statistical analysis, it is concluded not to be statistically significant and is of little clinical significance. As these studies confirm, external apical root resorption is an inevitable consequence of orthodontic tooth movement, no matter whether the tooth is treated with endodontics or with vital pulp.

Although most articles qualified for this systematic review indicated no significant differences in root resorption between vital and endodontically treated teeth, 4 studies reported that vital teeth underwent significantly greater external root resorption as compared to their contralateral root-filled teeth. Kurnaz and Buyukcavus observed the mean root length of vital teeth being $0.93 \pm 1.21 \mathrm{~mm}$ (non-extraction group) and 0.98 $\pm 1.18 \mathrm{~mm}$ (extraction group) shorter than endodontically treated teeth and stated these differences as statistically significant [17]. Khan, et al. study, variations in mean root length after orthodontic treatment between vital and root-filled teeth were less evident $(1.18 \pm 0.71$ $\mathrm{mm}$ and $0.85 \pm 0.68 \mathrm{~mm}$, respectively) although the differences remained statistically considerable [5]. Other studies carried out by Kolcuoğlu, et al. and Lee, et al. were in agreement with studies mentioned above and stated that significantly greater frequency of external root resorption was observed in vital teeth $[18,19]$. The reasons of these findings are questionable, considering the multifactorial etiology of root resorption [20]. However, the direction of tooth movement in addition to the magnitude and type of orthodontic force can be regarded as critical factors governing the amount of root resorption. All studies involved in this systematic review used the bracket system for orthodontic treatment, which ensured the continuous type of force. 
Nevertheless, the direction of tooth movement and the magnitude of orthodontic force were presented only in one study [18]. The lack of this information in other articles impeded the proper comparison between studies, therefore, it could be considered as the limitation. Since the positive correlations between increased force levels and increased root resorption has been confirmed [21], it is believed that excessive orthodontic forces enhance the expression of macrophage colony-stimulating factor (M-CSF), receptor activator of nuclear factor kappa-B ligand (RANKL), interleukin-1 $\beta$ (IL-1 $\beta$ ), and tumor necrosis factor alpha (TNF- $\alpha$ ) in the pulp cells via stretch-activated channels and result in odontoclastic inflammatory root resorption [22]. It is noteworthy that this mechanism can be observed only in vital teeth since endodontically treated teeth are devoid of pulp tissue and factors associated with root resorption are unable to be secreted in root-filled teeth without pulp. This could be the reason why four studies from our systematic review found significantly less resorption in endodontically treated teeth as compared to other six articles demonstrating no considerable differences between root-filled and vital teeth.

The results of this systematic review should be interpreted with caution due to the methodological limitations of involved studies. In addition to the limitations mentioned above, there was no possibility of subject allocation as only patients with the requirement of orthodontic treatment and having root-filled teeth with their contralateral vital teeth were included in all researches. Further, none of the studies included in the systemic review mentioned the materials used during endodontic treatment. Moreover, the root resorption was assessed by using different types of medical imaging techniques. 6 out of 10 studies used panoramic radiographs, which are considered to be less accurate than periapical radiographs [23]. It is believed that CBCT is the most effective method for evaluating the amount of root resorption in clinical basis [24], however, this evaluation method was chosen only in study by Castro, et al. One more study carried out by Kolcuoğlu and $\mathrm{Oz}$ used the micro-CT, which is generally regarded to be more accurate than the CBCT [25]. Nevertheless, the micro-CT can be used only for extracted teeth, therefore, pre-orthodontic evaluation has to be performed by another method, which can provide discrepancies by comparing values measured before and after orthodontic treatment. In addition, all studies, except Kolcuoğlu and $\mathrm{Oz}$, assessed external root resorption only in the apical third by measuring the tooth axial length. It is believed that this type of external root resorption in orthodontically treated teeth is more frequent and more severe in comparison with others [26]. However, external root resorption can occur in any third or surface of the root according to the direction of tooth movement. Finally, it is noteworthy that selecting contralateral homologous teeth as a control group remains the possibility of asymmetric ortho- dontic biomechanics regarding the different position of teeth.

\section{Conclusions}

Within the limitations of this systematic review, it can be concluded that endodontically treated teeth are affected by orthodontically induced external root resorption less or equally to vital teeth. Therefore, root- filled teeth can be safely involved in orthodontic treatment. It is necessary to mention that all teeth, no matter their vitality, show some degree of external root resorption during orthodontic tooth movement, therefore, it would be recommended to use tracking of the patients teeth by taking radiographs. Early detection could help to prevent severe complications and ensure a successful orthodontic treatment.

\section{Acknowledgment}

The authors have no funding and no conflicts of interest.

\section{References}

1. Nigam M, Tyagi S, Tiwari M, Mapare S, Zulfikar MM, et al. (2016) An insight to root resorption. Pre Clin Dent Res 2: 1-6.

2. Weltman B, Vig KW, Fields HW, Shanker S, Kaizar EE (2010) Root resorption associated with orthodontic tooth movement: A systematic review. Am J Orthod Dentofacial Orthop 137: 462-476.

3. Brezniak N, Wasserstein A (2002) Orthodontically induced inflammatory root resorption. Part I: The basic science aspects. Angle Orthod 72: 175-179.

4. Wahab RMA, Shafiai NAA, Ariffin SHZ (2017) An insight into risk factors for root resorption during orthodontic treatment. J Med Sci 17: 1-9.

5. Khan AR, Fida M, Shaikh A (2018) Evaluation of apical root resorption in endodontically treated and vital teeth in adult orthodontic subjects. J Ayub Med Coll Abbottabad 30: 506-510.

6. Llamas-Carreras JM, Amarilla A, Solano E, Velasco-Ortega E, Rodriguez-Varo L, et al. (2010) Study of external root resorption during orthodontic treatment in root filled teeth compared with their contralateral teeth with vital pulps. Int Endod J 43: 654-662.

7. Mah R, Holland GR, Pehowich E (1996) Periapical changes after orthodontic movement of root-filled ferret canines. J Endod 22: 298-230.

8. Gordon JM, Rosenblatt M, Witmans M, Carey JP, Giseon $\mathrm{Heo}$, et al. (2009) Rapid palatal expansion effects on nasal airway dimensions as measured by acoustic rhinometry: $A$ systematic review. Angle Orthod 79: 1000-1007.

9. Steadman SR (1942) Résumé of the literature on root resorption. Angle Orthod 12: 28-38.

10. Wickwire NA, Mc Neil MH, Norton LA, Duell RC (1974) The effects of tooth movement upon endodontically treated teeth. Angle Orthod 44: 235-242.

11. Andreasen JO, Andreasen FM (1992) Root resorption following traumatic dental injuries. Proc Finn Dent Soc 88: 95-114.

12. Esteves T, Ramos AL, Pereira CM, Hidalgo MM (2006) Orthodontic root resorption of endodontically treated teeth. $\mathrm{J}$ Endod 33: 119-122. 
13. Esteves T, Ramos AL, Hidalgo MM (2013) Apical root resorption of vital and endodontically treated teeth after orthodontic treatment: A radiographic evaluation. Dental Press Endod 3: 69-73.

14. Llamas Carreras JM, Amarilla A, Espinar Escalona E, Castellanos Cosano L, Martín González J, et al. (2011) External apical root resorption in maxillary root-filled incisors after orthodontic treatment: A split-mouth design study. Med Oral Patol Oral Cir Bucal 17: e523-e527.

15. Castro I, Valladares Neto J, Estrela C (2015) Contribution of cone beam computed tomography to the detection of apical root resorption after orthodontic treatment in root-filled and vital teeth. Angle Orthod 85: 771-776.

16. Giovanni HGI, Eliezer GL (2019) A comparison of the degree of external root resorption between endodontically treated and its vital contra lateral teeth after orthodontic treatment. Int J Fam Commun Med 3: 27-31.

17. Kurnaz S, Buyukcavus MH (2020) External root resorption in root-filled and vital teeth after extraction and non-extraction orthodontic treatments: A split-mouth retrospective study. Acta Odontol Scand 10:1-7.

18. Kolcuoğlu K, Oz AZ (2020) Comparison of orthodontic root resorption of root-filled and vital teeth using micro-computed tomography. Angle Orthod 90: 56-62.

19. Lee YJ, Lee TY (2016) External root resorption during orthodontic treatment in root-filled teeth and contralateral teeth with vital pulp: A clinical study of contributing factors. Am J Orthod Dentofacial Orthop 149: 84-91.

20. Dindaroğlu F, Doğan S (2016) Root Resorption in orthodontics. Turk J Orthod 29: 103-108.

21. Roscoe MG, Meira JB, Cattaneo PM (2015) Association of orthodontic force system and root resorption: A systematic review. Am J Orthod Dentofacial Orthop 147: 610-626.

22. Kaku M, Sumi H, Shikata H, Kojima S, Motokawa M, et al. (2014) Effects of pulpectomy on the amount of root resorption during orthodontic tooth movement. J Endod 40: 372378.

23. Pandis N, Nasika M, Polychronopoulou A, Eliades T (2008) External apical root resorption in patients treated with conventional and self-ligating brackets. Am J Orthod Dentofacial Orthop 134: 646-651.

24. Almeida Alves Costa PA, Oliveira Salgado I, Campos CN, Galdino TM (2011) External apical root resorption. Revista Interdisciplinar de Estudos Experimentais 3: 38-43.

25. Versiani M (2019) Studying root canal anatomy with non-destructive imaging tools. News Medical.

26. Feller L, Khammissa RA, Thomadakis G, Fourie J, Lemmer $J(2016)$ Apical external root resorption and repair in orthodontic tooth movement: Biological events. Biomed Res Int. 